\title{
Depression Risk in Young Adults: Could a Dopamine Receptor Gene Moderate the Role of Childhood Adversities?
}

\author{
Courtney Landers
}

\section{Abstract}

The mood disorder 'major depression' is a significant contributor to disability in society. One of the most significant determinants of an individual's risk of depression is their experience of adversities during childhood. Evidence is beginning to accumulate for a gene $\mathrm{x}$ environment relationship between childhood environment, including adversities, and genotype for the DRD4exIII-VNTR. The presence of a 7rpt allele for this polymorphism has been linked to increased sensitivity of externalising behaviours, pro-social behaviour and altruism to childhood environment. A recent study has found that the sensitivity of an individual's behavioural activation system - a cortical circuit that controls motivation and reaction to reward - is protected from adversities in childhood by this 7rpt allele. In this paper, a known negative correlation between the behavioural activation system and depression led to the hypothesis that DRD4 may also play a protective role against childhood adversities in terms of the risk of depressive symptoms. However, in a representative sample of the population $(\mathrm{n}=1,630)$ aged $20-24, D R D 4$ and childhood adversity were not found to interact in the formation of depressive symptoms $(\beta=0.069, p=0.381)$.

\section{Introduction}

Major depression is a mood disorder that is fast becoming a major issue in public health. The World Health Organization (WHO) rates depression as the fourth leading cause of disability worldwide, predicting it will become the number two cause - behind only obesity and diabetes-related disorders - by 2025 (Sapolsky, 2009). Depression is characterised by psychological symptoms, including anhedonia (inability to feel pleasure) (Beevers \& Meyer, 2002; Sapolsky, 2009) and lowered motivation towards reward (Pinto-Meza et al., 2006), neuroticism, negative affectivity and guilt (Roseman \& Rodgers, 2006; Sapolsky, 2009), 
along with physical symptoms such as psychomotor retardation and vegetative symptoms. All symptoms tend to occur in rhythmic patterns (Hasler et al., 2010; Sapolsky, 2009).

A well-established determinant of an individual's risk of major depression is the experience of adversity in childhood, including neglect, abuse, conflict and tension within a household along with parental alcohol and drug abuse or mental ill health (Rosenman \& Rodgers, 2004). Childhood adversities (CA) make a significant contribution to a society's mental health burden in general (Scott, Varghese and McGrath, 2010) via an association with the first onset (Green et al., 2010) and persistence (McLaughlin et al., 2010) of mental illness, particularly mood disorders. Although no links have been found between particular types of CA and specific mental illnesses (Green et al., 2010; Kessler, Davis \& Kendler, 1997), CA have been shown to increase risk of depression (Korkeila et al., 2005; Korkeila et al., 2010) and its onset during adolescence (Hazel et al., 2008). Variation in the strength of this predictive relationship across individuals raises the question of whether a gene $\mathrm{x}$ environment relationship may be involved.

The study of gene $x$ environment (GxE) interactions is undergoing a change in the way phenotypes are approached and measured. A bias towards investigation of negative environmental factors and negative individual outcomes has favoured the advance of the diathesis-stress model of GxE interactions, where particular genotypes increase the vulnerability of individuals to negative events (Belsky et al., 2009; Belsky, Bakermans-Kranenburg \& van Ijzendoorn, 2007). Recent research has suggested that the differential-susceptibility model proposed by Belsky (1997) may be more accurate. Proponents of this model argue that during human evolution, positive selection would have favoured the production of offspring that differed in their reactivity to the environment. Although the majority of environments would have been moderate (where extremes in behaviour would have been disadvantageous), challenging environments that required more extreme behaviours would have arisen regularly enough that it would be necessary for survival to produce offspring with varying reactivity (Belsky et al., 2007; Belsky et al., 2009; Boyce \& Ellis, 2005). A proposed example of the consequences of this adaptation in the modern world is the interaction of childhood environment with individual genotypes for the dopamine receptor D4 (DRD4).

Dopamine in the brain acts in a neuromodulative manner; rather than mediating short-term action potentials, it changes neuronal structure and function over the long-term (Rondou, Haegerman \& Van Craenenbroeck, 2010). In this way, dopamine is involved with motor output, endocrine function, attention, memory and learning. The current study focuses on the consequences of dopamine's role in motivation and reward response (Rondou et al., 2010). Dopamine acts via specific receptors such as DRD4, a member of the D2 family of dopamine 
receptors which is involved in the modulation of adenylyl cyclase, and thus the catalysation of cAMP production, via coupling to G proteins (Rondou et al., 2010). The significance of $D R D 4$ to GxE interactions stems from the existence of a variable number of tandem repeats (VNTR) polymorphism in exon three of the $D R D 4$ gene - the $D R D 4$-exIII-VNTR genotype. Globally, the most common allele of this polymorphism is the 4-repeat (4rpt) allele (64\% prevalent), followed by the $7 \mathrm{rpt}(21 \%)$ and $2 \mathrm{rpt}(8 \%)$ alleles, though these figures differ across populations (Chang et al., 1996). Evolutionary studies indicate the 7rpt allele is younger than the $4 \mathrm{rpt}$ allele, having increased rapidly in frequency due to strong positive selection despite the association of the allele with lower DRD4 expression, lower G-coupling potential and reduced second messenger generation (Ebstein et al., 2006; Rondou et al., 2010).

Recent research suggests the 7rpt allele conveys differential susceptibility to $\mathrm{CA}$ in the form of parenting style. The level of externalising behaviours opposition, overaction, aggression and attention (Bakermans-Kranenburg et al., 2008; Propper et al., 2007) - shown by toddlers with different DRD4-exIIIVNTR alleles appears to vary with maternal sensitivity. Children with one or more 7rpt alleles (+7rpt) showed greater behavioural responses to maternal sensitivity than children without a 7rpt allele (-7rpt) with the same exposure to maternal sensitivity (Bakermans-Kranenburg \& van Ijzendoorn, 2006; Bakermans-Kranenburg \& van Ijzendoorn, 2007). Specifically, +7rpt children with insensitive mothers showed more externalising behaviours than -7rpt children, while $+7 \mathrm{rpt}$ children with sensitive mothers showed less externalising behaviours than -7rpt children. The same pattern of results has been observed for interaction of sensation-seeking in toddlers with parenting quality (Sheese et al., 2007). Furthermore, when maternal sensitivity in parenting was improved using a specially-designed intervention program, +7rpt children showed the largest reduction in externalising behaviours, especially when their mothers showed the greatest improvements in sensitivity (Bakermans-Kranenburg et al., 2008). Similar findings have emerged recently, linking parenting, the 7rpt allele and positive, beneficial behaviours. The security of a toddler's attachment to his or her mother was found to affect altruistic behaviour - more secure children behaving more altruistically, and less secure children behaving less altruistically - but only in the presence of the 7rpt allele; in its absence, attachment and altruism showed no interaction (Bakermans-Kranenburg \& van Ijzendoorn, 2011). Likewise, parenting was found to influence 'prosocial behaviour', but only in +7rpt children (Knafo, Israel \& Ebstein, 2011). Thus there is mounting, correlating evidence that the 7rpt allele is a differential-susceptibility allele, increasing the reactivity of individuals possessing it to the quality of parenting they receive. This theory is further supported by the absence of main effects between the DRD4 gene and any of the behaviours investigated. Absence of such main effects is required for genes to fit the differential-susceptibility model 
(Belsky et al., 2007; Belsky et al., 2009). If, by making individuals more reactive to childhood adversities, the DRD4 7rpt allele can influence the development and display of dopamine-related traits such as externalisation and sensation seeking, it follows that the 7rpt allele could create variation in the relationship between CA and risk of major depression. Indeed, a possible connection has already been found between a dopamine-related personality measure with strong links to major depression - the behavioural activation scale - and the DRD4-exIII-VNTR x CA interaction.

The Behavioural Activation System (BAS) and Behavioural Inhibition System (BIS) were described by Gray $(1972 ; 1981)$ as a means of providing a biological basis of personality, and from that, behaviour. In their simplest forms, BIS and BAS are cortical circuits which stably and consistently determine how an individual will respond to cues of punishment and reward respectively (Gray, 1972; Gray, 1981; Carver \& White, 1994). The BAS in particular controls and produces positive feelings when reward occurs, as well as the motivation to move towards rewarding goals (Campbell-Sills, Liverant \& Brown, 2004; Kasch et al., 2002), thus underlying learning, emotion and motivation (Campbell-Sills et al., 2004). The strength of an individual's BAS is most commonly measured using Carver and White's (1994) BAS scale (a corresponding, intersecting scale exists for BIS). This scale has been directly designed to predict an individual's responses to events with an attached element of reward, scoring them within three dimensions determined and confirmed by factor analysis - drive, funseeking and reward responsiveness (Carver \& White, 1994; Jorm et al., 1999). Direct testing and experimental use has shown that this design successfully produces a measure of personality rather than mood (Campbell-Sills et al., 2004; Gomez \& Gomez, 2002; Jorm et al., 1999). This is an important distinction, given that personality is a much more stable and reliable predictor of behaviour than mood (Campbell-Sills et al., 2004; Gomez \& Gomez, 2002; Jorm et al., 1999). BAS scores nonetheless correlate with measures of positive affect (Carver \& White, 1994; Campbell-Sills et al., 2004). This relationship, plus correlations with positive processing of emotional stimuli and symptoms of anhedonia (Beevers \& Meyer, 2002; Campbell-Sills et al., 2004; Gomez \& Gomez, 2002) could mediate what appears to be a strong relationship between BAS scores and depression.

Depressed individuals have consistently been found to have lower BAS scores than non-depressed controls (Kasch et al., 2002; Campbell-Sills et al., 2004; Pinto-Meza et al., 2006). Furthermore, these BAS scores remain stable over diagnosis; entering remission from an episode of major depression does not alter an individual's BAS score (Kasch et al., 2002; Campbell-Sills et al., 2004; PintoMeza et al., 2006). In fact, BAS scores predict the progression of a depressive episode over a six-month period (McFarland et al., 2006). However, BAS scores have not yet been compared before and after the first onset of major depression. 
There is thus no indication yet of whether a low BAS score is a predictor of major depression, or a 'scar' signifying a profound change in personality caused by onset of depression (Kasch et al., 2002); Pinto-Meza et al., 2006). Despite this knowledge gap, it is clear that a strong, stable relationship exists between BAS and major depression.

Given a recent finding based on data from the Pathways Through Life (PATH) study that the presence of the DRD4 7rpt allele results in higher BAS scores across individuals who have experienced the same level of CA (Das et al., 2011), the relationship between BAS and depression provides a possible pathway for explaining variation in the effect of CA on depressive risk. In short, it appears a GxE interaction between DRD4-exIII-VNTR genotype and CA may moderate depressive risk via moderation of BAS. Investigating this pathway will involve examining each of the interactions involved, as well as determining whether DRD4-exIII-VNTR genotype x CA may moderate depressive risk without the intermediate of BAS. This investigation used multiple linear regressions within the PATH dataset to answer three key questions. First, can the protection of BAS from CA by the DRD4 7rpt allele be confirmed? Second, can the relationship between BAS and depression be confirmed within the PATH dataset? Finally, is there an interaction between DRD4-exIII-VNTR genotype, CA and depression, and if so is this independent of BAS, or is BAS a critical mediator?

\section{Methods}

\section{Participants}

The sample was taken from the PATH Through Life Project: a longitudinal study of three groups (20-24, 40-44 and 60-64 at baseline) of individuals randomly chosen from the electoral roll of Canberra and Queanbeyan, aimed at investigating mental health and ageing through surveys every four years for 20 years (Anstey et al., 2011). Participants contributed buccal epithelial cells for genotyping and were surveyed on many variables including experience of childhood adversities (included only in the first wave of the survey), personality, health and access to services (Anstey et al., 2011). This investigation used data from the first wave (Wave 1 ) of surveys in the +20 age cohort, comprised of 2,404 individuals aged 20-24. Only individuals reporting to be of European descent and with a particular DRD4-exIII-VNTR genotype (see below) were used in the investigation; individuals with missing values for any variables of interest were also excluded from the investigation. Final sample size was $n=1,630$. 


\section{Measures}

Depression was measured using the Goldberg Depression Scale (Goldberg et al., 1988), a two-part survey designed to quickly assess the occurrence of depressive symptoms in the past month. Scores range between 0 and 9; these were used as a continuous measure of depressive symptoms, without categorisation (Butterworth, Rodgers and Windsor, 2009).

CA were measured using a questionnaire designed by Rosenman and Rodgers (2004). This questionnaire measures experience of 17 different adversities including neglect, abuse, conflict and tension within a household along with parental alcohol and drug abuse or mental ill health - up to the age of 16 . Responses were summed, without weighting any items, to create a continuous variable. Evidence associating particular adversities with risk of mental illness, specifically depression, is as yet inconclusive (Green et al., 2010; McLaughlin et al., 2010); analysis was conducted using the CA score as a whole, without grouping any adversities (Das et al., 2011).

Experience of stressful life events (SLE) within the past six months was measured using the 12-item List of Threatening Experiences (Brugha et al., 1985). Sensitivity of the behavioural activation system was measured using the three BAS subscales designed by Carver and White (1994). Each subscale was used as an individual, continuous variable. The PATH dataset contains 10 different alleles and 25 different genotypes for the DRD4-exIII-VNTR polymorphism. Functional status has only been determined for the 4rpt and 7rpt alleles; incorporation of other, rare alleles without known functional properties into phenotypic analysis has been shown to confound results (Das et al., 2011). For this reason, only individuals with 4/4, 4/7 and 7/7 genotypes were included in the analysis.

\section{Statistical Analysis}

Data analysis was performed using SPSS18 (Chicago: SPSS Inc.). Multiple linear regressions were performed controlling for age, gender and total years of education in all models. Given strong evidence for correlation between experience of SLE and depression (Korkeila et al., 2005; Korkeila et al., 2010), individual experience of SLE within the last six months was also controlled for in all models. Regression models were generated by setting outcome variables, then entering covariates, followed by predictors and then, if applicable, an interaction term. For each model, $R^{\wedge} 2$ values and change in $R^{\wedge} 2$ values from previous steps were noted, along with beta and $\mathrm{p}$ values. The first round of regressions was performed using the BAS subscales - Drive, Fun seeking and Reward response - as outcome variables with each subscale tested in separate 
models. The predictors were DRD4-exIII-VNTR genotype, number of CA experienced and an interaction variable for $D R D 4$-exIII-VNTR genotype and CA. DRD4-exIII-VNTR genotype was entered into the models as a binary categorical predictor; the $4 / 4$ genotype was classified as -7 and the $4 / 7$ and $7 / 7$ genotypes were pooled into a +7 category, due to the rarity of the $7 / 7$ genotype (Das et al., 2011). Although CA were observed in a range of 0-14, few individuals reported more than 5 adversities; scores equal to or greater than 5 were pooled to create a scale from 0 to 5 where $0=$ no adversities and $5=$ five or more adversities. A second round of regressions tested whether the BAS subscales were significant predictors of depression. Each BAS subscore was tested as a predictor for depression within its own regression. A third round of regressions then investigated whether DRD4-exIII-VNTR genotype, number of CA experienced and an interaction variable for $D R D 4 \times \mathrm{CA}$ significantly predicted depressive symptoms.

\section{Results}

Sociodemographic characteristics of this sample are reported in Table 1. Sociodemographic variables, number of CA and SLE, and mean Goldberg scores were not significantly different between 7+ and 7- groups. Hardy Weinberg Equilibrium could not be directly confirmed within the dataset used but Das et al. (2011) report that the distribution of genotypes within the PATH dataset is not significantly different from Hardy Weinberg Equilibrium.

Regression models (using the same controls) were generated for possible correlation between depressive symptoms (as outcome) and BAS scores, revealing a significant association (Table 2). Greater scores for depressive symptoms were negatively associated with lower scores for all three BAS subscales.

A series of linear regressions (controlled for age, total years of education, gender and number of SLE experienced in the past six months) were used to investigate whether BAS scores were significantly predicted by DRD4-exIIIVNTR genotype, number of CA experienced and an interaction term for the gene x environment relationship between $D R D 4$-exIII-VNTR genotype and CA. Genotype showed no significant prediction of BAS scores. However both CA and the interaction term predicted one or more of the BAS subscores (Table 3). Lower scores on the BAS Reward subscale were correlated with a larger number of reported CA. The interaction term was negatively correlated with both BAS Drive and Reward subscores. In contrast to the result of Das et al. (2011), this suggests that the presence of the 7rpt allele is sensitising to the effects of CA on BAS, rather than protective. 
The ANU Undergraduate Research Journal

Table 1. Demographic characteristics, adversities and depression measures of individuals with 7+ and 7-DRD4-exonlII-VNTR genotypes (Mean \pm s.d. for continuous variables and frequency for categorical variables shown).

\begin{tabular}{|c|c|c|c|c|c|c|}
\hline & & \multicolumn{2}{|c|}{ DRD4-exonIII-VNTR genotype } & \multirow[t]{2}{*}{$t / \chi^{2}$} & \multirow[t]{2}{*}{ df } & \multirow[t]{2}{*}{$\mathbf{p}$} \\
\hline & & $7-(n=985)$ & $7+(n=645)$ & & & \\
\hline \multirow[t]{3}{*}{$\operatorname{sex}$} & & & & 0.276 & 1 & 0.599 \\
\hline & Male & $471(59.8 \%)$ & $317(40.2 \%)$ & & & \\
\hline & Female & $514(61.0 \%)$ & $328(39.0 \%)$ & & & \\
\hline age & & $22.55 \pm 1.5$ & $22.59 \pm 1.5$ & 0.445 & 1628 & 0.657 \\
\hline education & (years) & $14.61 \pm 1.6$ & $14.48 \pm 1.6$ & -1.624 & 1621 & 0.105 \\
\hline \multirow[t]{7}{*}{$\mathrm{CA}$} & & & & 7.141 & 5 & 0.210 \\
\hline & 0 & $416(59.1 \%)$ & $288(40.9 \%)$ & & & \\
\hline & 1 & $220(58.4 \%)$ & $157(41.6 \%)$ & & & \\
\hline & 2 & $120(65.9 \%)$ & $62(34.1 \%)$ & & & \\
\hline & 3 & $75(65.2 \%)$ & $40(34.8 \%)$ & & & \\
\hline & 4 & $60(67.4 \%)$ & $29(32.6 \%)$ & & & \\
\hline & $\geq 5$ & $85(57.0 \%)$ & $64(43.0 \%)$ & & & \\
\hline \multirow[t]{7}{*}{ SLE } & & & & 8.598 & 5 & 0.126 \\
\hline & 0 & $265(60.6 \%)$ & $172(39.4 \%)$ & & & \\
\hline & 1 & $239(56.9 \%)$ & $181(43.1 \%)$ & & & \\
\hline & 2 & $212(64.8 \%)$ & $115(35.2 \%)$ & & & \\
\hline & 3 & $107(58.2 \%)$ & $77(41.8 \%)$ & & & \\
\hline & 4 & $56(56.0 \%)$ & $44(44.0 \%)$ & & & \\
\hline & $\geq 5$ & $97(66.9 \%)$ & $48(33.1 \%)$ & & & \\
\hline Goldberg & & $2.98 \pm 2.4$ & $2.86 \pm 2.4$ & -0.978 & 1619 & 0.328 \\
\hline
\end{tabular}

$\mathrm{t}$ tests were performed for continuous variables and $\square^{2}$ tests for categorical variables CA childhood adversity

SLE stressful life events experienced in past six months

Goldberg Goldberg Depression Scale

Table 2. Multiple linear regression models with each Behavioural Activation System subscore predicting depression

\begin{tabular}{llll}
\hline Predictors & $\boldsymbol{\beta}$ & $\mathbf{p}$ & $\mathbf{R}^{2}$ (change) \\
\hline BAS & & & \\
drive & -0.127 & $\mathbf{0 . 0 0 0}$ & $0.166(0.015)$ \\
reward response & -0.075 & $\mathbf{0 . 0 0 0}$ & $0.153(0.015)$ \\
fun seeking & -0.072 & $\mathbf{0 . 0 0 0}$ & $0.153(0.005)$ \\
\hline
\end{tabular}

All models controlled for age, gender, total years of education and number of stressful life events experienced in the past six months

$\mathrm{p}<0.05$ shown in bold

BAS Behavioural Activation System 
Table 3. Multiple linear regression models for interactive effect of DRD4-exIIIVNTR genotype and childhood adversities on the Behavioural Activation System

\begin{tabular}{|c|c|c|c|c|c|c|c|c|c|}
\hline \multirow[t]{2}{*}{ Predictors } & \multicolumn{3}{|c|}{ BAS-drive } & \multicolumn{3}{|c|}{ BAS-fun seeking } & \multicolumn{3}{|c|}{ BAS-reward } \\
\hline & $\boldsymbol{\beta}$ & $\mathbf{p}$ & $\begin{array}{l}\mathrm{R}^{2} \\
\text { (change) }\end{array}$ & $\boldsymbol{\beta}$ & $\mathbf{p}$ & $\begin{array}{l}\text { R2 } \\
\text { (change) }\end{array}$ & $\boldsymbol{\beta}$ & $\mathbf{p}$ & $\begin{array}{l}R^{2} \\
\text { (change) }\end{array}$ \\
\hline $\begin{array}{l}\text { DRD4-exIII- } \\
\text { VNTR }\end{array}$ & -0.018 & 0.477 & $\begin{array}{l}0.015 \\
(0.000)\end{array}$ & 0.017 & 0.487 & $\begin{array}{l}0.035 \\
(0.000)\end{array}$ & -0.033 & 0.186 & $\begin{array}{l}0.007 \\
(0.000)\end{array}$ \\
\hline $\mathrm{CA}$ & -0.032 & 0.135 & $\begin{array}{l}0.015 \\
(0.000)\end{array}$ & -0.023 & 0.274 & $\begin{array}{l}0.040 \\
(0.000)\end{array}$ & -0.045 & 0.035 & $\begin{array}{l}0.012 \\
(0.001)\end{array}$ \\
\hline CA x DRD4 & -0.201 & 0.022 & $\begin{array}{l}0.018 \\
(0.003)\end{array}$ & -0.074 & 0.395 & $\begin{array}{l}0.036 \\
(-0.001)\end{array}$ & -0.179 & 0.041 & $\begin{array}{l}0.010 \\
(0.002)\end{array}$ \\
\hline
\end{tabular}

All models controlled for gender, age, total years of education and the number of stressful life events experienced in the last six months

$\mathrm{p}<0.05$ shown in bold

CA childhood adversity

BAS behavioural activation system

The final regression model used $D R D 4$-exIII-VNTR genotype, number of CA experienced and the DRD4-exIII-VNTR genotype x CA interaction variable as predictors for depressive symptoms. While CA showed significant associations with depressive symptoms, DRD4-exonIII-VNTR genotype and the DRD4-exIIIVNTR genotype x CA interaction variable did not (Table 4). A significant main effect was found between CA and depressive symptoms, with larger numbers of reported CA predicting higher levels of depressive symptoms; however, no significant main effect was found between depressive symptoms and genotype for DRD4-exIII-VNTR. There was then no significant interaction of the DRD4exIII-VNTR x CA interaction term with depressive symptoms. This strongly suggests that DRD4-exIII-VNTR genotype does not moderate the effect of CA on risk of depressive symptoms in the same way it moderates the impact of CA on BAS scores.

Table 4. Multiple linear regression models with DRD4-exIII-VNTR genotype, childhood adversities and an interaction variable predicting depression

\begin{tabular}{llll}
\hline Predictors & $\boldsymbol{\beta}$ & $\mathbf{p}$ & $\mathbf{R}^{\mathbf{2}}$ (change) \\
\hline DRD4-exIII-VNTR & 0.019 & 0.404 & $0.174(-0.001)$ \\
CA & 0.191 & $\mathbf{0 . 0 0 0}$ & $0.179(0.033)$ \\
CA x DRD4 & 0.069 & 0.381 & $0.210(0.000)$ \\
\hline
\end{tabular}

All models controlled for age, gender, total years of education and number of stressful life events experienced in the past six months

$\mathrm{p}<0.05$ shown in bold

CA childhood adversity 


\section{Discussion}

This investigation has confirmed the previous finding of a relationship between individual sensitivity of the behavioural activation system and depression, but has not confirmed a previously found relationship between the BAS, experience of CA and genotype for the DRD4-exonIII-VNTR polymorphism. It also failed to find an interaction between CA, DRD4-exIII-VNTR genotype and depression. These results have interesting implications and suggest directions for future research.

The findings of this investigation confirm the existence of a negative correlation between individual BAS scores and depression (Kasch et al., 2002; CampbellSills et al., 2004; Pinto-Meza et al., 2006). Given that the BAS has a biological basis - i.e. it is associated with particular brain centres, the development and operation of which will affect behavioural activation - this association has the potential to assist elucidation of the biological basis of depression, including its genetic links. Before this can occur, the nature of the BAS-depression relationship needs to be determined. Not only could the PATH dataset be used to confirm the stability of BAS scores over depression states (Kasch et al., 2002; Campbell-Sills et al., 2004; Pinto-Meza et al., 2006), it contains longitudinal data that could be used to investigate whether BAS scores change or remain constant over the first onset of depression. The question of whether low BAS scores are a 'scar' of depression or a contributing cause (Kasch et al., 2002) is an important one - can depression permanently alter personality, or does our personality predispose us to depression? Tackling this question will help direct further research into what mediates the BAS-depression relationship. Anhedonia and rhythmic patterns are both symptoms of depression (Sapolsky, 2009); both have also been suggested as links to BAS. Beevers and Meyer (2008) found that a significant association of BAS with positive experiences, which were significantly associated with positive expectations, mediated a significant negative association between BAS and anhedonic symptoms of depression. A relationship between morningnesseveningness (individual patterns in alertness and activity over a day) and depression also appears to correlate with BAS via positive affect; a daily rhythm in behavioural activation, and thus in positive affect, has been suggested as an explanation (Hasler et al., 2010).

This investigation also confirms that within the PATH dataset, CA and DRD4exIII-VNTR genotype interact to affect BAS Drive and Reward subscores. However the interaction found here is negative. Thus instead of supporting the theory proposed by Das et al., (2011) of a protective role for DRD4-exIII-VNTR genotype in mediating a main effect between CA and these BAS subscores, it suggests a sensitising role, or possibly a differential susceptibility role like the one DRD4-exIII-VNTR genotype appears to play between childhood 
environment and externalising behaviours, altruism and prosocial behaviour. The explanation for this conflict may be two variations in method between this investigation and that of Das et al. (2011). First, the baseline group used was different; while this investigation used data only from wave 1 of the +20 group, Das et al. (2011) used +20 data up to and including wave 3 . The first onset of depression is known to often occur in early adulthood (Hazel et al., 2008), so it is possible that personality changes occurred within the PATH sample between waves 1,2 and 3 of the $20+$ group. Second, the coding of the DRD4exIII-VNTR genotype variable and thus the $D R D 4$-exIII-VNTR genotype x CA interaction variable was conducted differently between these two investigations (Das, personal communication); this has statistical implications that could also explain the differing results. The exact reasons behind the conflict are worth investigating; it is likely however that given a few adjustments in method, the analysis conducted in this investigation would confirm the finding of Das et al. (2011). The task for future research will then be the explanation of this relationship, which will first involve demonstrating the same interaction within other datasets. Determining the psychological and biological basis of this relationship could then be complicated by the contrast between the role DRD4-exIII-VNTR genotype plays between CA and BAS, compared to its role between CA and other forms of behaviour. Previous studies have found the presence of the 7rpt allele appears to increase an individual's susceptibility to both positive and negative environments, in the form of parenting, resulting in respectively more positive or negative outcomes in terms of externalising and prosocial behaviour (Bakermans-Kranenburg and van Ijzendoorn, 2006; Bakermans-Kranenburg and van Ijzendoorn, 2007; Bakermans-Kranenburg et al., 2008; Bakermans-Kranenburg and van Ijzendoorn, 2011; Knafo et al., 2011). In this case however, the 7rpt allele is protective against increasingly negative environments; the 'complementary' role in a differential-susceptibility context would be to decrease the positive affects of increasingly positive environments on behaviour, an unlikely occurrence. How can the same allele play a differential-susceptibility and protective role in mediating the effects of very similar stressors on behavioural traits closely linked via their connections to motivation, reward and thus dopamine (Rondou et al., 2010)?

Despite confirmation of correlations between BAS and depressive symptoms and between a DRD4-exIII-VNTR genotype x CA interaction and BAS (even with possible conflict over the nature of those interactions), no correlation between the DRD4-exIII-VNTR genotype x CA interaction and depression was found, even when BAS subscores were not controlled for. To some degree, this is expected. The DRD4 allele has not been linked to depression before. The polymorphism most discussed in relation to gene $\mathrm{x}$ environment moderation of depression is the serotonin transporter polymorphism 5 - HTTLPR (Chipman et al., 2007). However, there are a number of relationships that are difficult 
to explain in the absence of a DRD4-exIII-VNTR genotype x CA interaction link to depression. As discussed earlier, the $D R D 4$-exIII-VNTR genotype x CA interaction appears to moderate those BAS subscores most closely linked to depression via positive affect and anhedonia - drive and reward. Furthermore, the investigation that first found the protective effect of DRD4-exIII-VNTR genotype on BAS from CA also found that the presence of the 7rpt allele protected individuals from a decrease in levels of resilience after experiencing CA (Das et al., 2011). Resilience levels are known to be one of the many determinants for risk of mental illness, particularly depression (Das et al., 2011). Finally, the connection between dopamine and depression via anhedonia, reward response and motivation (discussed in Introduction) cannot be ignored (Rondou et al., 2010; Sapolsky, 2009).

\section{Limitations and Confounds}

It is possible that the different findings of this investigation compared to the previous investigation by Das et al. (2011) could be due to its design. The most common limitation for analysis based on self-questionnaire data is the problem of biased recall (Korkeila et al., 2005; Korkeila et al., 2010; Rosenman and Rodgers, 2004). A recent investigation into the relationship between the reasons by which individuals with depressive symptoms and those around them understand their illness, and the 'objective' causes behind those depressive symptoms, suggests that self-report questionnaires may not accurately capture individual risk factors for depression. Kendler, Myers and Halberstadt (2011) found that individuals who had experienced SLE that made their illness 'understandable' to themselves and others were just as likely to have clinical (other illnesses), environmental (e.g. child abuse), genetic or personality factors that 'explained' their depression as individuals with depression who lacked experience of recent trauma. In other words, external stressors are not sole explanations for depressive symptoms, but individuals with depression and those around them may treat these events as such. This raises the question of whether individuals may fail to record other possible causes for their depression in self-questionnaires, which would disguise the underlying cause for a perhaps substantial portion of depression cases, confounding the analysis of investigations such as this one. Given that the results of Kendler et al. (2011) are correct, equally as confounding is why stressful life events are still found to be such strong predictors of depression. Perhaps those individuals with reasons for their illness are more likely to report their symptoms, and individuals without reasons more likely to feel their symptoms are unjustifiable and unexplainable, and fail to report them. Controlling for SLE when including CA in an analysis is common practice due to their demonstrated link to depression independently and additively with CA (Hazel et al., 2008; Korkeila et al., 2005; Korkeila et al., 2010). Yet in the situation discussed above this may remove from analysis both variability caused by factors other than 
SLE, and those individuals most likely to report depressive symptoms, leaving a sample size that is too small to capture gene $\mathrm{x}$ environment interactions with mental illness.

Even if the reporting of SLE did not bias the reporting and analysis of other causes for depressive symptoms, SLE still present other potential limitations. First, attempting to analyse the effects of solely CA on depression presents the challenge of controlling not only for a main effect between SLE and depression, but also a complex interactive effect of CA and SLE; CA may lead to SLE due to sensitisation to trauma and predisposition to adult risk factors (Korkeila et al., 2005; Korkeila et al., 2010). Second, the PATH dataset only contains records of SLE experienced by participants in the last six months before the survey occurred, an interval that has been suggested as too short both to capture all SLE which may be contributing to depressive symptoms and for SLE to begin to affect occurrence of depressive symptoms (Chipman et al., 2007).

The strong comorbidity of depression with other mental illnesses (Kessler et al., 1997) could also have confounded these findings. Given that the first onset of depression is often during adolescence (Hazel et al., 2008) the use of the +20 age group from the PATH dataset should have helped ensure that individuals experiencing depressive symptoms had not yet developed symptoms of other mental illnesses. However, this age bracket does not exclude individuals who may be experiencing depressive symptoms comorbid to mental illness that onset in early adolesence or childhood, such as Attention Deficit Hyperactive Disorder (ADHD).

Comorbidity of ADHD with depression may explain the failure of this investigation to find an association between the DRD4-exIII-VNTR genotype $\mathrm{x}$ CA interaction and depression. Symptoms of depression have been found to be comorbid with ADHD in individuals between 18 and 44 years of age (Kessler et al., 2006), and several studies, including meta-analyses, have found a small but significant association between ADHD and the DRD4 7rpt allele (Faraone et al., 1999; Faraone et al., 2000; Muglia et al., 2000). A possible explanation may be that DRD4-exIII-VNTR genotype mediates the effect of CA only in depression occurring comorbid to ADHD and thus that the age group analysed was too young to contain enough individuals with symptoms of comorbid depression and ADHD. A further explanation could be that the DRD4-exIIIVNTR genotype $\mathrm{x}$ CA interaction does not associate directly with depression at all but associates with ADHD instead, and through comorbidity to ADHD, with other mental illnesses.

The final limitations on this investigation are those inherent in the PATH Through Life dataset. There are significant advantages in using this dataset. First, the use of random selection from the electoral roll for participant recruitment 
provides a more community-based, unbiased sample than would be obtained if individuals were recruited via hospitals, GPs and other institutions. Second, the inclusion of Goldberg's Depression Scale in the surveys administered to PATH participants also provides data on sub-clinical levels of depression, allowing analysis of the full spectrum of depressive symptoms and how genes and the environment interact to influence that spectrum. However, as the symptoms of depression and other mental illnesses recorded in the PATH dataset are not clinically diagnosed (instead measured using questionnaires) this makes them, and any results based on them, less reliable than if these symptoms had been clinically diagnosed.

\section{Conclusion}

No interaction between CA, DRD4-exIII-VNTR genotype and depression was found in this study, and a relationship previously found between BAS, CA and DRD4-exonIII-VNTR genotype was not confirmed. However, this investigation contributes to observations of a relationship between an individual's BAS score and depression.

\section{Acknowledgements}

This study was approved by the ethics committee of the Australian National University. All participants gave informed written consent to be included in the PATH study. The author is grateful to Simon Easteal and Debjani Das for their supervision of this investigation and to Susan Tan for her work in providing the genotype data required for the investigation. The author thanks Dr Moyra Mortby and Professor Mauricio Arcos-Burgos for critical review of the manuscript.

\section{References}

Anstey KJ, Christensen H, Butterworth P, et al.: Cohort Profile: The PATH through life project. International journal of epidemiology, 2011.

Bakermans-Kranenburg MJ, van Ijzendoorn MH: Gene-environment interaction of the dopamine D4 receptor (DRD4) and observed maternal insensitivity predicting externalizing behavior in preschoolers. Developmental Psychobiology 48:406-409, 2006. 
Bakermans-Kranenburg MJ, Van IMH, Pijlman FT, et al.: Experimental evidence for differential susceptibility: dopamine D4 receptor polymorphism (DRD4 VNTR) moderates intervention effects on toddlers' externalizing behavior in a randomized controlled trial. Developmental Psychobiology 44:293-300, 2008.

Bakermans-Kranenburg MJ, van Ijzendoorn MH: Differential susceptibility to rearing environment depending on dopamine-related genes: new evidence and a meta-analysis. Developmental Psychobiology 23:39-52, 2011.

Beevers CG, Meyer B: Lack of positive experiences and positive expectancies mediate the relationship between BAS responsiveness and depression. Cognition and Emotion 16:549 - 564, 2002.

Belsky J: Variation in susceptibility to environmental influence: An evolutionary argument. Psychological Inquiry 8182 - 186, 1997.

Belsky J, Bakermans-Kranenburg MJ, van Ijzendoorn MH: For better and for worse: Differential susceptibility to environmental influences. Current Directions in Psychological Science 16:300 - 304, 2007.

Belsky J, Jonassaint C, Pluess M, et al.: Vulnerability genes or plasticity genes? Molecular Psychiatry 14:746-754, 2009.

Boyce WT, Ellis BJ: Biological sensitivity to context: I. An evolutionarydevelopmental theory of the origins and functions of stress reactivity. Development and Psychopathology 17:271-301, 2005.

Brugha T, Bebbington P, Tennant C, et al.: The List of Threatening Experiences: a subset of 12 life event categories with considerable long-term contextual threat. Psychological Medicine 15:189-194, 1985.

Butterworth P, Rodgers B, Windsor TD: Financial hardship, socio-economic position and depression: results from the PATH Through Life Survey. Social Science and Medicine 69:229-237, 2009.

Campbell-Sills L, Liverant GI, Brown TA: Psychometric evaluation of the behavioral inhibition/behavioral activation scales in a large sample of outpatients with anxiety and mood disorders. Psychol Assess 16:244-254, 2004.

Carver CS, White TL: Behavioral inhibition, behavioral activation, and affective responses to impending reward and punishment. Journal of Personality and Social Psychology 67:319-333, 1994.

Chang FM, Kidd JR, Livak KJ, et al.: The world-wide distribution of allele frequencies at the human dopamine D4 receptor locus. Human Genetics 98:91-101, 1996. 
Chipman P, Jorm AF, Prior M, et al.: No interaction between the serotonin transporter polymorphism (5-HTTLPR) and childhood adversity or recent stressful life events on symptoms of depression: results from two community surveys. American Journal of Medical Genetics B Neuropsychiatric Genetetics 144B:561-565, 2007.

Das D, Cherbuin N, Tan X, et al.: DRD4-exonIII-VNTR moderates the effect of childhood adversities on emotional resilience in young-adults. PLoS One 6:e20177, 2011.

Ebstein RP: The molecular genetic architecture of human personality: beyond self-report questionnaires. Molecular Psychiatry 11:427-445, 2006.

Gomez R, Corr PJ: Attention-Deficit/Hyperactivity Disorder symptoms: Associations with Gray's and Tellegen's models of personality. Personality and Individual Differences 49:902-906, 2010.

Green JG, McLaughlin KA, Berglund PA, et al.: Childhood adversities and adult psychiatric disorders in the national comorbidity survey replication I: associations with first onset of DSM-IV disorders. Archives of General Psychiatry 67:113-123, 2010.

Hasler BP, Allen JJB, Sbarra DA, et al.: Morningness-eveningness and depression: preliminary evidence for the role of the behavioral activation system and positive affect. Psychiatry Research 176:166-173, 2010.

Hazel NA, Hammen C, Brennan PA, et al.: Early childhood adversity and adolescent depression: the mediating role of continued stress. Psychological Medicine 38:581-589, 2008.

Jorm AF, Christensen H, Henderson AS, et al.: Using the BIS/BAS scales to measure behavioural inhibition and behavioural activation: Factor structure, validity and norms in a large community sample. Personality and Individual Differences 26, 1999.

Kasch KL, Rottenberg J, Arnow BA, et al.: Behavioral activation and inhibition systems and the severity and course of depression. Journal of Abnormal Psychology 111:589-597, 2002a.

Kasch KL, Rottenberg J, Arnow BA, et al.: Behavioral activation and inhibition systems and the severity and course of depression. J Abnorm Psychol 111:589-597, 2002b.

Kendler KS, Myers J, Halberstadt LJ: Do reasons for major depression act as causes? Molecular Psychiatry 16:626-633, 2011. 
Kessler RC, Davis CG, Kendler KS: Childhood adversity and adult psychiatric disorder in the US National Comorbidity Survey. Psychological Medicine 27:1101-1119, 1997.

Kessler RC, Adler L, Barkley R, et al.: The prevalence and correlates of adult ADHD in the United States: results from the National Comorbidity Survey Replication. The American Journal of Psychiatry 163:716-723, 2006.

Knafo A, Israel S, Ebstein RP: Heritability of children's prosocial behavior and differential susceptibility to parenting by variation in the dopamine receptor D4 gene. Development and Psychopathology 23:53-67, 2011.

Korkeila J, Vahtera J, Nabi H, et al.: Childhood adversities, adulthood life events and depression. Journal of Affective Disorders 127:130-138, 2010.

Korkeila K, Korkeila J, Vahtera J, et al.: Childhood adversities, adult risk factors and depressiveness: a population study. Social Psychiatry and Psychiatric Epidemiology 40:700-706, 2005.

McFarland BR, Shankman SA, Tenke CE, et al.: Behavioral activation system deficits predict the six-month course of depression. Journal of Affective Disorders 91:229-234, 2006.

McLaughlin KA, Green JG, Gruber MJ, et al.: Childhood adversities and adult psychiatric disorders in the national comorbidity survey replication II: associations with persistence of DSM-IV disorders. Archives of General Psychiatry 67:124-132, 2010.

Muglia P, Jain U, Macciardi F, et al.: Adult attention deficit hyperactivity disorder and the dopamine D4 receptor gene. American Journal of Medical Genetics 96:273-277, 2000.

Pinto-Meza A, Caseras X, Soler J, et al.: Behavioural inhibition and behavioural activation systems in current and recovered major depression participants. Personality and Individual differences 40, 2006.

Propper C, Willoughby M, Halpern CT, et al.: Parenting quality, DRD4, and the prediction of externalizing and internalizing behaviors in early childhood. Developmental Psychobiology 49:619-632, 2007.

Rondou P, Haegeman G, Van Craenenbroeck K: The dopamine D4 receptor: biochemical and signalling properties. Cell and Molecular Life Sciences 67:1971-1986, 2010.

Rosenman S, Rodgers B: Childhood adversity in an Australian population. Social Psychiatry and Psychiatric Epidemiology 39:695-702, 2004. 
Rosenman S, Rodgers B: Childhood adversity and adult personality. Australian New Zealand Journal of Psychiatry 40:482-490, 2006.

Scott J, Varghese D, McGrath J: As the twig is bent, the tree inclines: adult mental health consequences of childhood adversity. Archives of General Psychiatry 67:111-112, 2010.

Sheese BE, Voelker PM, Rothbart MK, et al.: Parenting quality interacts with genetic variation in dopamine receptor D4 to influence temperament in early childhood. Developmental Psychopathology 19:1039-1046, 2007. 